\title{
The Role of Enhanced Recovery in Robotic Cystectomy
}

\author{
Goonewardene SS ${ }^{1 *}$, Persad R ${ }^{2}$, Gillatt $\mathrm{D}^{3}$ \\ ${ }^{1}$ University of Warwick, Guys and St Thomas Hospitals London \\ ${ }^{2}$ North Bristol NHS Trust, Bristol \\ ${ }^{3}$ Mcquarrie University, Sydney
}

Received: February 01, 2016; Accepted: February 22, 2016; Published: March 24, 2016

*Corresponding author: Goonewardene SS, University of Warwick, Guys and St Thomas Hospitals London, Email: ssg7727@yahoo.co.uk

\section{Dear Editor,}

There have been a number of articles recently examining pain within enhanced recovery $[1,2]$. This clearly highlighted the patients enhanced recovery pathway after open surgery. It has a number of factors contributing to their recovery [1]. This included significantly fewer opioid analgesics, possibly contributing to decreased postoperative ileus and shorter length of hospital stay. In today's' world, the focus seems to be on robotic surgery as the driving force behind shorter recovery periods and day case surgery [3]. However, appropriate patient selection may be true in prostate surgery; the same cannot be said for robotic cystectomy. With a far wider resectional area and greater stress response, appropriate patient selection beforehand and support afterwards is a key to good patient outcomes.

Enhanced Recovery After Surgery (ERAS) studies have shown objective parameters that can improve peri-op outcomes [4]. However, a survey has shown significant individual differences in peri-op management of bladder cancer with the majority of urologists using personal experience as a primary guide [4].

The key to successful postoperative recovery with robotic cystectomy is preoperative, intraoperative, and postoperative care advances with an implementation of cystectomy enhanced recovery pathways [5]. This is a novel way to reduce length of stay and overall complications within 90 days of cystectomy without increasing readmissions or complications as demonstrated by $\mathrm{Xu}$, et al [2]. A well-designed pathway in combination with robotic surgery can dramatically improve complications and length of stay compared to the national standards [6].

The pathway starts pre-operatively, with an assessment of the patient using cardiopulmonary exercise testing. The use of cardiopulmonary exercise testing is gaining popularity as a preoperative functional assessment tool and is a useful adjunct to risk stratification before radical cystectomy [7].

The enhanced recovery pathway implements a series of evidence-based interventions that decreases the length of stay and complications without compromising patient outcomes [5]. A standard pathway incorporates preoperative education, expectation setting, rehabilitation, nutrition evaluation, carbohydrate loading, venous thrombosis prophylaxis, normothermia maintenance, local anaesthesia, no nasogastric tubes or bowel prep, early feeding, and opioid avoidance [5].

Robotics alone is not enough to maximise patient outcome, the benefits of enhanced recovery are also required with robotic cystectomy.

\section{References}

1. Xu W, Ahmadi H, Cai J, Miranda G, Shuckman A, Daneshmand S, et al. Post-operative pain management after radical cystectomy: Comparing traditional and enhanced recovery after surgery protocol at USC. AUA. 2015.

2. Xu W, Daneshmand S, Bazargani ST, Cai J, Miranda G, Schuckman AK, et al. Postoperative Pain Management after Radical Cystectomy: Comparing Traditional versus Enhanced Recovery Protocol Pathway. J Urol. 2015;194(5):1209-1213.

3. Goonewardene SS, Rowe E. Radical robotic assisted laparoscopic prostatectomy: A day case procedure. BJU International. 2014;24(11):804-805. doi:10.1089/lap.2014.0468.

4. Truong H, Nix J, Smith K, Mittal A, Agarwal P. Perioperative management of radical cystectomy patients: A questionnaire survey of the American Urological Association members. J Clin Oncol. 2013;suppl 6.

5. Kukreja JB, Kiernan M, Schempp B, Hontar A, Ghazi A, Rashid H, et al. Cystectomy enhanced recovery pathway: Reduction in length of stay without increased morbidity or readmissions. J Urol. 2015;19(4):813814 .

6. Athanasiadis G, Soares R, Swinn M, Perry M, Jones C, Patil K. Setting up a new robot assisted radical cystectomy service. European Urology Supplements. 2014;13(3):31.

7. Hoyland K, Vasdev N, Adshead JM, Thorpe A. Cardiopulmonary exercise testing in patients undergoing radical cystectomy (open, laparoscopic and robotic). Journal of Clinical Urology. 2014;7(6):374379. doi: 10.1177/2051415814537823.

Citation: Goonewardene SS, Persad R, Gillatt D (2016) The Role of Enhanced Recovery in Robotic Cystectomy. Int J Adv Robot Automn 1(1): 1. 